Instituto Internacional de Investigación y Desarrollo Tecnológico Educativo INDTEC, C.A.

DOI: https://doi.org/10.29394/Scientific.issn.2542-2987.2019.4.12.19.351-368

OAI-PMH: http://www.indteca.com/ojs/index.php/Revista Scientific/oai

Ensayo Original / Original Essay

\title{
Las Prácticas Profesionales como Escenario de Aprendizaje para Desarrollar Procesos Cognitivos de Alto Nivel
}

Autores: Rosilio Ramón Alfonzo Mendoza Universidad Nacional Experimental "Rafael María Baralt", UNERMB rosilioalfonzo@gmail.com Trujillo, Venezuela https://orcid.org/0000-0002-0430-850X

Yizza María Delgado Nery De Vita Universidad Nacional Experimental "Rafael María Baralt", UNERMB yizzadelgadodevita@gmail.com Trujillo, Venezuela https://orcid.org/0000-0001-9193-7453

\section{Resumen}

El ensayo mostrado presenta como objetivo, analizar los procesos cognitivos de alto nivel, que intervienen en la enseñanza de los estudiantes de prácticas profesionales. El estudio fue abordado, donde el ensayista incluye teorías de interés analizadas de forma referencial, práctica y reflexiva sobre el tema. Haciendo uso de los conocimientos analíticos planteados por Carr y Kemmis (1986); y Tallaferro (2006). De manera conclusiva, se resalta: las prácticas profesionales como espacio de aprendizaje llegan a permitir adquirir y desarrollar habilidades en la profesión sustentadas en el "saber hacer" y debe hacerse durante toda la carrera para lograr el alcance deseado; se observan más los compendios negativos que los positivos, disminuyendo expectativas en el posterior trabajo como enseñante, el profesor transmite más información, que el potenciamiento eficaz en los términos cognitivos, capaces de admitir en los estudiantes el resaltar dificultades cognitivas y resolver problemas, en la cotidianidad.

Palabras clave: enseñanza; ambiente educacional; cognición. 


\title{
Professional Practices as a Learning Scenario to Develop High Level Cognitive Processes
}

\begin{abstract}
The aim of this essay is to analyze the high level cognitive processes that intervene in the teaching of students of professional practices. The study was addressed, where the essayist includes theories of interest analyzed in a referential, practical and reflective manner on the subject. Making use of the analytical knowledge proposed by Carr and Kemmis (1986); and Tallaferro (2006). In a conclusive way, it is highlighted: professional practices as a learning space come to allow acquiring and developing skills in the profession based on "know-how" and must be done throughout the career to achieve the desired scope; Negative compendiums are observed more than positive ones, diminishing expectations in the later work as a teacher, the teacher transmits more information, than effective empowerment in cognitive terms, able to admit students to highlight cognitive difficulties and solve problems, in the everydayness.
\end{abstract}

Keywords: teaching; educational environment; cognition. 


\section{Planteamiento Introductorio}

Ahondar en argumentos iniciales para un proceso investigativo, es comunicar concepciones, motivaciones y prácticas acerca de aquello que inquieta, interroga y que simultáneamente, provoca diferentes maneras de conocimiento. Por esto, el objetivo propuesto es analizar los procesos cognitivos de alto nivel, que intervienen en la enseñanza de los estudiantes de prácticas profesionales. De hecho, cuando se reflexiona sobre la temática que se elige, aunado con el modo como pedagogo de prácticas profesionales, donde el investigador reflexiona acerca de la pasión, que se tiene por la ciencia debatiendo interrogantes, que ha estado pensando hace tiempo.

Más aún, son relevantes los docentes universitarios, que imparten prácticas profesionales, tomando en cuenta su experiencia, van construyendo saberes propios que se desean explorar en este ensayo, no como fin de apropiarse de éstos, sino para buscar la posibilidad de relacionarlos con otros y así, educarse de él observando diferencias.

Así, las universidades asumen una dinámica responsabilidad en cuanto, a los procesos formativos estudiantiles, en sus prácticas profesionales. Por ende, con una individualización y determinación epistemológica respecto, al progreso en los procesos cognoscitivos de alto nivel, como formas de posesionarse del conocimiento, y en particular el discernimiento pedagógico, con miras a identificar los conocimientos de cambio en los futuros docentes, actualmente formándose.

La situación descrita, posee de una visión posmoderna, donde se pueda apreciar el tiempo, tomando en consideración el elemento social inmerso en lo educativo, académico, donde la práctica profesional sea el eje fundamental. En palabras de Gimeno y Pérez (2008): "la práctica profesional es un elemento medular del currículo en la formación inicial universitaria. La misma ha sido considerada, inclusive, como una corriente, enfoque u orientación curricular" (pág. 31). Desde esta perspectiva, el acto formativo es visto como tendencia 
curricular en espacios de estática y dinamismo dando lugar a comprender los elementos estructurantes que la conforman, aunado a, recomendaciones instituidas entre ellos.

Sin embargo, se insiste que la práctica profesional aplica bajo componentes propios brindados por la misma realidad contextual, respecto a hechos o contextos prácticos presentados en cada dinámica. Asimismo, la teoría para esta visión compone el intelecto, capaz de descifrar realidades, además, de aludir certezas imprevistas capaces de una constante verificación.

Por esto, insignes pedagogos como Simón Rodríguez, Jesualdo Iglesias y Paulo Freire, han dado aportes a la pedagogía educativa cuyos postulados, siguen vigentes en este momento. A tal efecto, la educación de Paulo Freire, según mi postura es la que ha tenido más impacto y desarrollo no sólo en Latinoamérica y América del Sur, pues sus categorías de análisis fueron la utopía, la analogía planteada entre teoría y práctica, el vínculo con la política, cualidades que en verdad reflejaban la compleja situación observada en los países latinoamericanos.

Asimismo, la indagación mostrada coincide cuando plantea la problemática entre lo teórico y lo práctico según Carr y Kemmis (1986):

El tipo de relación entre «medios» y «fines» que presupone el enfoque positivista de lo teórico y lo práctico no sirve para entender cómo, en la educación, las finalidades, las políticas y los métodos están intrínsecamente relacionados. En particular, al considerar que las cuestiones de valor sólo pueden referirse a los fines, ignora la peculiar relación que existe entre los fines de la educación (pág. 158).

Dicho de otra manera, el profesor tutor de prácticas profesionales deberá entender que para obtener cientificidad, como método educativo significa limitar las hipótesis empíricas generadas, utilizando recursos pedagógicos con mayor eficacia. No obstante, resulta propio no descuidar esa limitante pues se estaría actuando bajo una concepción simplista educativa y 
además, es ignorar la condición en los medios educativos, reflejando siempre valores educacionales. Infiriendo entonces, es imposible eliminar estas valoraciones y reducir a lo instrumental las cuestiones acerca de éstos porque sería, en último análisis, conseguir que éstos desertaran definitivamente, de ser educativos.

Sintetizando lo expuesto, el precio de la teoría radica en su capacidad para ilustrar el pensamiento de los enseñantes. Es decir, la dialéctica teoríapráctica reposa para que la hipótesis facilite vías para el examen crítico de las experiencias pedagógicas prácticas. Sin embargo, la supuesta fundamentalidad acerca del final de la teoría educativa y su dualidad con la práctica continúan todavía incólumes.

Asimismo, se insiste que la práctica en los educandos próximos a ser profesionales deberá aplicarse bajo elementos propios que la misma situación o contenido, ofrece en función de cada destreza. Asimismo, la hipótesis para esta perspectiva representa un alcance, como medio para explicar lo real, que constituye verdades inciertas que demandan una continua justificación.

Igualmente, en Venezuela hoy se concibe con fuerza optimar y transformar la instrucción, propuestas de formación que incumben al contexto pedagógico. Las nuevas tendencias, sugieren especialmente, a los organismos universitarios, la responsabilidad de constituir docentes íntegros, con capacidad de obtener los beneficios que en ésta se otorgan.

De hecho, se observan problemáticas en los establecimientos universitarios porque no se hace una revisión honorable, a mi forma de pensar administrativa y pedagógica donde se inserten nuevas directrices, basadas en un currículo innovador, diferentes planes de estudio acordes a necesidades contextuales, a fin de obtener una instrucción avocada a aspectos integrales donde de sapiencias nuevas con las ya adquiridas por los estudiantes, en su devenir académico. No obstante, entendiendo la decepción observada en las prácticas profesionales, suponen distanciamiento, desconexión entre teoría y 
práctica, porque la práctica se realiza en la última etapa del currículo pedagógico.

Es decir, cuando ya el estudiante dispone del discernimiento probado y sus normas, aconteciendo asiduamente al culminar la carrera. De hecho, debe haber preocupación por Prácticas Profesionales donde se observe un equilibrio entre teoría/práctica, ofreciendo oportunidades para analizar, sintetizar y reflexionar teóricamente.

Así, se alude lo expuesto por Edeltstein (2003): donde "las prácticas estén sustentadas en diversas explicaciones de los procesos de enseñar y aprender en relación con los sujetos, el conocimiento y las condiciones institucionales en que se concretan" (pág. 20); las prácticas profesionales se deben reconocer como interrogantes para desarrollar conceptualizaciones, que puedan devengar futuras investigaciones, enfocadas desde la praxis del futuro docente profesional.

Todo esto, es dificultoso de abreviar porque, es el resultado de un agregado de variables coincidentes, que llevarán probablemente a una alineación indeleble que parece decantarse. Atendiendo a esta perspectiva, avizoran momentos de crisis, anarquía y desconcierto, donde el profesor deba solventar conflictos de distinta índole que acontecen. Imaginando un permisible mañana, acompañado de un deseable acontecimiento en la formación del educando.

Cabe considerar, la preocupación sobre la Práctica Profesional desplegada por los estudiantes que se constituyen como docentes en los recintos universitarios, se encuentra confinada bajo perspectivas que ocupan el ambiente de enseñanza o ámbito formativo, parece que ha faltado entendimiento y ha caído en el error de situarla en una representación única, imparcial aplicando aisladamente, separada de toda mediación social en los diferentes contextos culturales.

Visto así desde el contexto educativo, las prácticas profesionales 
percibidas como escenario de aprendizaje, para optimar los procesos cognitivos, son esenciales las aulas educativas, pues el docente deberá conocer el potencial y las capacidades innatas en sus estudiantes, en cuanto, al desarrollo de los procesos cognitivos. Así se alude por cognición lo expuesto por Parkin (1999), citado por Nieto (2015): "es la rama de la psicología que intenta proporcionar una explicación científica de cómo el cerebro lleva a cabo funciones mentales complejas como la visión, la memoria, el lenguaje y el pensamiento" (pág. 43); de esta forma, lo referente a la cognición implica el estudio de los procesos cognitivos básicos y superiores responsables de la superioridad de nuevos conocimientos.

Dicho de otra manera, el estudiante tiene que desplegar procesos cognitivos a través del ejercicio, prácticas de actividades, para esto el docente deberá calificar o comentar sugerencias ocurridas por su accionar educativo; referente al trabajo en grupo, deberá incentivarlos para que sean capaces de resolver problemáticas en su entorno. Además, utilizar la meditación sobre los contenidos curriculares y luego utilizar la comprensión para darles la utilidad pertinente; formular interrogantes respecto a los nuevos conocimientos adquiridos, cómo ha cambiado su visión, respecto a un tema, preguntas dirigidas a que tengan curiosidad, quieran saber.

Estos argumentos expuestos, obtienen trascendencia porque en este momento, se dibujan nuevos roles para las universidades, los docentes y la educación habitual, pues ese papel acostumbrado otorgado al profesor en el pasado, hoy es transformado y respaldado por un mediador en el trascurso del conocimiento, centrado en la instrucción de los estudiantes. Asimismo, fortalecer el progreso de procesos cognitivos, capacidades, actitudes, valores y propuestas curriculares, cónsonas con las pretensiones del futuro y adecuadas socialmente.

Por consiguiente, en el pensamiento intervienen procesos complejos rápidos inconscientes y tan fugaces que impiden memorizar. De hecho, los 
intentos de explicar los procesos cognitivos tienen lugar, y esto sigue significando uno de los grandes desafíos del ser humano. No obstante, abordar estos procesos cognitivos, es prioridad en todo contexto educativo, donde se está formando el futuro profesional que tendrá que enfrentar cambios que reclaman resolver problemas coherente y creativamente.

Así, para el citado ensayo, se objeta la cognición de los procesos psicológicos superiores, referentes a: tomar decisiones, proceso precedido por deliberaciones, para elegir diversas opciones, para ello se adoptan herramientas con objetividad y así alcanzar certezas en las decisiones. Resolución de problemas, busca fórmulas a resolver, habilidades para recordar dificultades similares, reconocer patrones y la creatividad. Sin embargo, hay posibilidades de no desarrollar con efectividad los conocimientos cognitivos de alto nivel antes mencionados, en el ejercicio educativo de los estudiantes.

\section{Desarrollo Argumentativo}

En el actual ensayo, se realizará la argumentación teórica que va a proporcionar la visión holística, que tienen las prácticas profesionales para lograr aprendizajes, referentes a procesos cognitivos superiores o de alto nivel. Además, se presenta la sustentación teórica del tópico a estudiar, lo que compondrá el soporte a la indagación, considerándose la relación con el estudio presentado y los argumentos significativos.

\subsection{Visión Teórica de las Prácticas Profesionales como Modelo de Formación}

La colectividad ubica habilidades individuales, herramientas formativas, donde los aprendices en distintas carreras, asuman su compromiso de un aprendizaje para toda la vida. Aprender y enseñar significa, ser un hecho fortuito ni de permanencia restringida, sino más bien es concebido como un 
asunto de toda la vida, según Tallaferro (2006a): "comienza cuando quien sueña con hacer de la docencia su vocación, recibe su primera clase en una institución de formación docente y culmina cuando la vida lo coloca por última vez frente a sus estudiantes" (pág. 3).

Esta postura de la autora, la comparto respecto a la vocación del docente que redundará en los aprendizajes, donde se posea la capacidad de transferir conocimientos a sus estudiantes, para que su transitar académico sea para provecho referente, a la asimilación de nuevos contenidos y experiencias significativas.

Partiendo de esta afirmación, en el quehacer educativo las prácticas dirigidas al proceso formativo son procesos duraderos toda la vida, no pueden ser vistas como algo ocasional, es como un manantial donde confluyen hábitos, culturas, escenarios, producidos en contextos diferentes e igualmente válidos.

Esto supone, que el practicante debe conceptualizar y al transcurrir del tiempo, las pueda adecuar como referente, en la forma propia de su cognición hacia su aprendizaje. Asimismo, elegir las prácticas pertinentes para aplicar al contexto inherente, porque sirve para el refuerzo del estudio presentado, dado que potencia en los educandos el progreso de las destrezas cognitivas básicas, a la vez mejora relaciones interpersonales y favorece para construir la episteme de éstos.

En concordancia con el texto anterior, el papel formativo de las prácticas constituye una vivencia, porque las prácticas no sólo son haceres, dado que esto implica normas, tradiciones, y accionar en el cosmos vivencial, es decir, también son prácticas sociales porque se muestra quién se es, cómo se conceptualiza vida y educación. Se denota, disociación en el deber ser, pues teoría y práctica no deben estar separadas, asumiendo el saber teórico como la epistemología propia de la experiencia, conocimientos, creándose una relación diferenciada y unidireccional de ambos elementos. 
Así, aludiendo lo expuesto, Tallaferro (2006b), establece: "señalar que ni a lo largo de su formación ni en el ejercicio de su profesión, encuentran los modos de vincular los referentes teóricos con el quehacer en las aulas" (pág. $5)$; en otras palabras, esta debilidad se pudiera percibir en algunos contextos educativos, con la introducción de prácticas ya estudiadas con anterioridad, que avalen una formación, para mejorar problemáticas y situaciones que afecten determinada comunidad, y la formación sea dirigida para satisfacer y solventar necesidades del ámbito circundante.

Por esto, las opiniones que planteó, pienso que son vitales y justifican asumir un compromiso responsable, como formadores de estudiantes, para que sean competentes de desarrollar durante su formación, la disposición reflexiva hacia desarrollar los procesos cognitivos superiores y la disposición, hacia el aprendizaje significativo.

\subsection{Acepciones de la Práctica Profesional: Miradas en el Tiempo y Retrospectiva hacia los Escenarios de Aprendizaje.}

Pareciera saber todo sobre el profesor y su formación, más poco se analiza, investiga y se escribe sobre esto, específicamente, como objeto a estudiar, como campo epistemológico. Así, se alude respecto al plano educativo, ante esto, Terán, Perdomo y Castillo (2017): afirman que "la educación busca la evolución, transformación e inmortalidad del ser humano; la enseñanza forma, prepara, desarrolla, perfecciona al hombre en su vida, en su convivencia social, lo ayuda a encontrarse, así mismo, a proyectarse ante los demás..." (págs. 31-32).

En otras palabras, educar para una enseñanza dirigida a proyectar al ser humano en la consecución de habilidades propias para su desempeño a lo largo de su existencia, es relevante en estos tiempos. Además, saber relacionar éstas en las prácticas profesionales, porque de ellas dependerá en la fusión de lo teórico con lo práctico realizar una simbiosis, que como deber 
ser deberá estar asociada a los diferentes contextos, como parte del sano convivir en todo medio social.

Partiendo de esta afirmación, las prácticas profesionales deben estar diseñadas para provocar en el educando, provecho por lo que enseña el profesor y por lo que él estudia, es decir ambos se deben ocupar por proyectar una instrucción académica óptima, donde el educador sea capaz de utilizar elementos para mejorar lo epistémico, a la vez, que fomenta una praxis donde la reflexión y forma de pensar sean fundamentales educativamente.

Sin embargo, en este momento, hay más preocupación por descubrir, tal como lo expresa Imbernón (2001): "cómo, de qué manera, con qué modelos, y, sobre todo, la conciencia de que ese conocimiento y esas aptitudes debían ser revisadas y actualizadas es algo mucho más reciente" (pág. 64); es decir, es actual reflexionar e indagar metodológicamente, sobre dicha formación del educador, inicial o permanente, hoy se nota más ocupación, en cuanto que sea ofrecida a entes que les concierne dicha postura.

En este panorama, se vincula todo un devenir hacia un auténtico cambio, porque se pretende formar profesionales responsables en el acontecer educativo, en los espacios universitarios, apoyado en el modelo racional técnico donde se observa un escalafón entre el conocimiento científico básico aplicado y las bifurcaciones sistemáticas de la práctica profesional. Sin duda, el mayor desacierto en la formación de los docentes es la distancia y desconexión entre la teoría y práctica, se observa que en el porcentaje sustantivo la práctica, está situada en la etapa conclusiva del currículo formativo.

Es decir, ésta sucede cuando el estudiante ya ha percibido un cúmulo de sapiencias científicas, en el último año de la carrera. En este caso, la práctica significa una acción, al aplicar procedimientos que se aprendieron teóricamente, permitiendo vinculaciones lógicas y rigurosas ante la situación 
desconocida.

Es evidente, que así no se desarrollan las prácticas correspondientes y relevantes a la argumentación, más es significativo el acercamiento de consideraciones teóricas, dado que interesa explorar y dilucidar las emanaciones, que germinan en las prácticas profesionales para el avance de los procesos cognoscentes superiores.

De manera similar, un aspecto relevante a considerar, cuando se realizan las prácticas profesionales es que éstas sean reflexivas, dirigidas a la enseñanza-aprendizaje, donde la misión del docente como formador es preparar profesores futuristas capaces de reflexionar sobre su praxis, concibiendo la práctica según Richardson (1994), citado en Gómez (2008): “la investigación sobre la práctica ha cambiado del foco en el comportamiento eficaz de los profesores a la comprensión de cómo los profesores construyen su comprensión del aprendizaje y la enseñanza" (pág. 31).

Sin embargo, hacer reflexión sobre la práctica significa hacer exploración, investigación para poder comprender acertadamente, las prácticas profesionales, y ahondar en aspectos que subyacen a éstas, donde la idea no es producir algo, sino más bien ampliar e instruir las probidades de profesores y estudiantes, a favor de brindar soluciones con las pertinentes condiciones y restricciones para hallar el camino adecuado. Algunos puntos clave sobre la mirada que expone De Vicente (1999), en torno al desarrollo de las prácticas como praxis reflexiva en la que:

Se necesita capacitar a los profesores para que se empeñen en procesos cognitivos de pensamiento reflexivo, para que adquieran la capacidad cognitiva y las creencias, valores, actitudes y emociones facilitadoras de tales procesos. Y si la persona tiene que empeñarse en un acto particular de reflexión, debe poseer la motivación necesaria para la reflexión (pág. 60).

Asimismo, Villar (1999), enfatiza: "Llegar a ser un profesor reflexivo significa e implica la probidad intelectual de utilizar procesos cognoscitivos de 
análisis interno de fenómenos curriculares, educativos. Incluye, además, la realización de actividades afectivas y morales en las aulas de los centros escolares..." (pág. 22). El profesor reflexivo debe poseer una mente abierta, con apertura mental y ser sincero, hacerse preguntas respecto a su accionar, interpretar resultados, comprometerse con las emanaciones, sin conformarse con los objetivos, sino que discute si las consecuencias son satisfactorias, y la deliberación la realiza mediante la acción.

No obstante, el investigador en su afán de cuestionar su investigación se pregunta: ¿Cómo es el deber ser en el reflexionar de las prácticas profesionales? Se piensa, que cada uno descubrirá su propio modo de hacerlo, si se recapacita que en su deber ser, es observar los fundamentos en que las sustenta, que conlleva a enfrentar resultados de su práctica. Desde ese accionar como educadores, debe haber el incentivo y ayudar a los estudiantes para que manifiesten preguntas como: ¿De qué manera desarrollo mi práctica?; ¿Es contradictorio el accionar de acuerdo con lo que digo o pienso?; ¿Cómo justifico las discrepancias entre principios y práctica?; ¿Qué debo cambiar?; ¿Qué y cómo he aprendido?; ¿Cómo refrescaría mi accionar en las prácticas profesionales?

La emanación de las premisas citadas con anterioridad, convida a revisar las experiencias educativas como responsabilidad insoslayable, que tienen quienes están abocados a ser formadores porque deben invadir espacios que propicien inquietudes, donde todos estemos llamados a la meditación, asumiendo que ésta, en las prácticas profesionales es la visión ontológica o forma del ser.

\subsection{Conjeturas de las Prácticas Profesionales como Escenario de Aprendizaje.}

Sin duda, la práctica pasa de ser un lugar de atención, a ejecutar las teorías aprendidas durante el transitar académico. Además, de ser un 
escenario donde se propicien aprendizajes teóricos, significando que los conocimientos, ideas, teorías educativas y pedagógicas formalicen un diálogo con las prácticas académicas del docente del proceso formativo. No obstante, se concibe como beneficio de una historia organizacional, que responde a cada escenario, caracterizándose por presentar variados argumentos tangibles e intangibles, siempre modelados por el tiempo. Se parte de la idea, que la práctica profesional es un componente esencial del currículo en la alineación originaria universitaria.

Por esto, en el ámbito universitario será conveniente aproximarnos a la generalidad de espacio, donde se aprecien cualidades intrínsecas de este término y cómo se emplean para tratados posteriores. Continuando, con las ilustradas palabras de Lupasco (1963): "los sistemas engendran sus espacios propios, como consecuencia de la simultaneidad de dinamismos antagónicos formadores, desarrollan sus tiempos propios de la sucesión dialéctica impuesta por la disyunción de estos dinamismos" (pág. 78).

Sintetizando, todo sistema procesa lugar y período, que le sirve para lograr los objetivos que se proponen, a la vez de relacionar, las prácticas profesionales con el estudio mostrado referido al escenario de aprendizaje, para beneficio en el progreso de sólidas aptitudes científicas, cognitivas, afectuosas.

\section{Aspectos Conclusivos}

Referente, a las prácticas profesionales como espacio de aprendizaje, llegan a permitir adquirir y desarrollar habilidades en la profesión sustentadas en el "saber hacer" y debe hacerse a lo largo de la carrera para lograr el alcance deseado. Es decir, el estudiante al desarrollar competencias se verá capacitado en el mundo laboral-profesional desde los inicios de la formación.

Habitualmente, se observan más los compendios negativos que los positivos, disminuyendo expectativas en el posterior trabajo como enseñante, 
también al ejecutar su rol encuentra insuficientes vínculos con el deber ser real que tiene que afrontar en el espacio universitario. Se contempla además, de manera concluyente, que el profesor transmite más información, que el potenciamiento eficaz en los conocimientos epistemológicos, es decir, esto indica que la acción docente ayuda muy poco a la edificación de herramientas cognitivas capaces de permitir a los alumnos resaltar dificultades y resolver problemas en la cotidianidad.

\section{Referencias}

Carr, W., \& Kemmis, S. (1986). Teoría Crítica de la Enseñanza. La investigación-Acción en la Formación del Profesorado. Cap. 5, 140166. Barcelona, España: Universidad de Arte y Ciencias Sociales. Recuperado de:

http://www.academia.edu/7360913/Wilfred Carr Stephen Kemmis 19 86 Teor\%C3\%ADa cr\%C3\%ADtica de la ense\%C3\%B1anza

De Vicente, P. (1999). La Formación del Profesorado como Práctica Reflexiva. En L. Villar. (coord). Un ciclo de enseñanza reflexiva. Estrategia para el diseño curricular. Bilbao, España: Ediciones Mensajero.

Edeltstein, G. (2003). Memoria, Experiencia, Horizontes... En Prácticas y Residencias. I Jornadas Nacionales de Prácticas y Residencias en la Formación de Docentes. Córdoba, España: Editorial Brujas, págs. 7189.

Gimeno, S., \& Pérez, Á. (2008). Comprender y Transformar la Enseñanza. España: Ediciones Morata.

Gómez, L. (2008). Los Determinantes de la Práctica Educativa. Universidades, (38), 29-39, ISSN: 0041-8935. Recuperado de:

https://www.redalyc.org/articulo.oa?id=37303804

Imbernón, F. (2001). Claves para una Nueva Formación del Profesorado. 
Investigación en la escuela, (43), 57-66, ISSN: 0213-7771, ISSN-e: 2443-9991. Recuperado de:

https://dialnet.unirioja.es/servlet/articulo?codigo $=117059$

Lupasco, S. (1963). Las Tres Materias. Buenos Aires, Argentina: Editorial Sudamericana.

Nieto, M. (2015). Análisis de los Procesos Cognitivos en el Alumnado de Enseñanzas Profesionales de Danza. Tesis Doctoral. Málaga, España: Universidad de Málaga. Recuperado de:

https://riuma.uma.es/xmlui/bitstream/handle/10630/11914/TD NIETO ROMERO Maria Macarena.pdf?sequence $=1$

Tallaferro, D. (2006a,b). La formación para la práctica reflexiva en las prácticas profesionales docentes. Educere: Revista Venezolana de Educación, (33), 269-273, ISSN-e: 1316-4910. Recuperado de:

https://dialnet.unirioja.es/servlet/articulo?codigo=2101104

Terán, C., Perdomo, E., \& Castillo, R. (2017). Un Recorrido por el Pensamiento Educativo hacia la Transformación de la Educación en el Siglo XXI. Revista Scientific, 2(5), 29-48. Recuperado de: https://doi.org/10.29394/scientific.issn.2542-2987.2017.2.5.2.29-48

Villar, L. (1999). Enseñanza Reflexiva. En L. Villar. (coord.). Un ciclo de enseñanza reflexiva. Estrategia para el diseño curricular. Bilbao, España: Ediciones Mensajero. 


\section{Rosilio Ramón Alfonzo Mendoza \\ e-mail rosilioalfonzo@gmail.com}

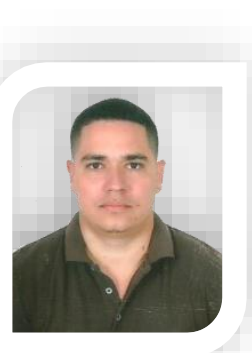

Nacido en Trujillo, Venezuela. Profesor ordinario, escalafón-asistente del área de prácticas profesionales del departamento de Ciencias Pedagógicas; Coordinador administrativo e investigador del grupo Geociencia y Taller "Abya-yala", línea de investigación Geohistoria; Asesor y/o jurado evaluador de trabajos de grado en pregrado y postgrado del Núcleo Universitario Rafael Rangel de la Universidad de Los Andes, Venezuela; Investigador activo del área de investigación histórica del Centro de Historia "Casa de los Tratados de Bolívar y Sucre", Trujillo, Venezuela; Coordinador del área de investigación histórica. 


\section{Yizza María Delgado Nery De Vita \\ e-mail: yizzadelgadodevita@gmail.com}

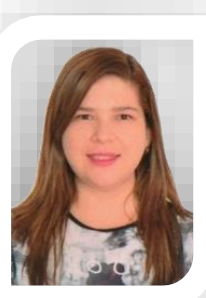

Nacida en estado Trujillo, Venezuela. Profesora ordinaria, escalafón-Asistente del área de Metodología de la Investigación del Departamento de Ciencias Sociales de la Universidad de Los Andes, Venezuela; Investigadora del Grupo en Estudios Críticos Culturales "Salvador Valero"; Miembro de la Comisión de Trabajos de grado de la carrera de Comunicación Social y miembro de la Comisión Curricular de la Carrera de Educación; Investigadora acreditada por el Observatorio Nacional de Ciencia y Tecnología; asesora y/o jurado evaluador de trabajos de grado en pregrado y postgrado. Por otra parte, tengo experiencia en producción radial, fotografía y uso de redes sociales para fines educativos.

El contenido de este manuscrito se difunde bajo una Licencia de Creative Commons ReconocimientoNoComercial-Compartirlgual 4.0 Internacional 